\title{
Morphometric evaluation and planning of anticurvature filing in roots of maxillary and mandibular molars
}

\section{Maria Antonieta Veloso Carvalho de OLIVEIRA(a) \\ Jessyca Figueira VENÂNCIO(a) Luís Henrique Araújo RAPOSO(b) Nelson BARBOSA JÚNIOR(c) João Carlos Gabrielli BIFFI(a)}

\footnotetext{
(a) Universidade Federal de Uberlândia UFU, School of Dentistry, Department of Endodontics, Uberlândia, MG, Brazil.

(b) Universidade Federal de Uberlândia UFU, School of Dentistry, Department of Occlusion, Fixed Prosthodontics and Dental Materials, Uberlândia, MG, Brazil.

(c) Universidade Federal de Uberlândia UFU, School of Dentistry, Department of Endodontics, Uberlândia, MG, Brazil.
}

Declaration of Interests: The authors certify that they have no commercial or associative interest that represents a conflict of interest in connection with the manuscript.

\section{Corresponding Author: João Carlos Gabrielli Biffi E-mail: jcgbiffi@ufu.br}

DOI: 10.1590/1807-3107BOR-2015.vol29.0012

Submitted: May 05, 2014 Accepted for publication: Aug 27, 2014 Last revision: Nov 04, 2014

\begin{abstract}
This study aimed to guide the planning of anticurvature filing using pre-determined anatomical points on teeth to establish directions for proper implementation of the technique. Two hundred digital periapical radiographs of human molar teeth were selected and divided into two groups ( $\mathrm{n}=100)$ : MX (maxillary) and MD (mandibular) molars. Mesiobuccal roots were considered for the MX group and mesial roots for the MD group. Pre-determined anatomical points required for planning the anticurvature filing on the root canal path were located, and the distances between these points obtained. The anticurvature filing was simulated in two different protocols for each group, and the region of dentin removal and the remaining dentin thickness were measured in the safety and danger zones of the root canals. Statistical analysis was carried out at a significance level of 5\%. The distances between the anatomical points and the thickness of remaining dentin showed significant differences when the two groups were compared $(p<0.001)$. No significant differences were found between the two experimental groups regarding the area of dentin removal at the root region, but differences were detected in comparison with dentin removal at the crown $(p<0.001)$. In terms of wear produced after simulation of both anticurvature filing protocols, significant differences were verified for all regions, except for the dentin remaining at the danger zone. The radiographic location of anatomical points allows for planning and implementation of controlled and efficient anticurvature filing and can be performed in the same manner for maxillary and mandibular molars.
\end{abstract}

Keywords: Endodontics; Molars; Root Canal Preparation.

\section{Introduction}

According to the crown-down technique, root canal preparation can be divided into three steps: coronal access, radicular access, and apical instrumentation. ${ }^{1}$ Coronal access is obtained with total removal of the root pulp chamber roof and proximal wall convexities. ${ }^{1,2}$ Radicular access, also known as anticurvature filing, should be carried out systematically in the molar roots. ${ }^{3}$

The enlargement of root canals preceding apical preparation has many advantages, such as eliminating dentin irregularities and providing better performance for instruments and irrigating agents in the apical region. ${ }^{1,4}$ Furthermore, the correct establishment of the working length is facilitated, ${ }^{5}$ which offers better control of the biomechanical preparation. ${ }^{6,7,8}$ 
The use of this technique also reduces the number of contaminants that can be extruded. ${ }^{9}$ Besides making the action of instruments more effective, with lower risk of fractures, since adequate access and space are ensured,$^{10}$ this technique also allows for better compaction of gutta-percha in the apical region. ${ }^{11}$

Conversely, overflaring of root canals should be avoided, because it promotes weakening of dental structure and can result in perforations, mainly on the furcal regions of teeth with curved root canals. ${ }^{12}$ The risk of perforation in areas with reduced dentin volume, considered danger zones, can be reduced by directed filing to areas of higher dentin volume, or safety zones, as recommended in the anticurvature filing. ${ }^{3}$ This technique has been used since the 1980s, first with hand instruments and Gates-Glidden drills and later with rotary instruments. ${ }^{10,12,13,14}$ However, recent studies have shown that there is no reduction in the perforation risk in danger zones (furcal region) when anticurvature filing is performed, even with Nickel-Titanium (NiTi) rotary systems. ${ }^{11,12}$

The excessive wear of coronal dentin also increases the risk of vertical root fractures in safety zones. ${ }^{11}$ The coronal chamber walls should be prepared only up to the extent necessary for the proper access of endodontic instruments. ${ }^{15}$ Maintenance of coronal and root dentin, especially in the cervical area, is essential to maximize the long-term results of the restorative procedures in endodontically treated teeth,$^{15}$ because the ability of a tooth to resist lateral forces is directly proportional to the dentin thickness between the canal walls and root surfaces. ${ }^{16}$

The literature recommends that the files should have straight direct access to the apical region after anticurvature filing. ${ }^{1,6,9,10,11}$ However, there are no studies confirming the possibility of obtaining straight direct access to all canals without the removal of excessive dentin structure in the safety zone of the root. Several investigations have been carried out on the danger zones in curved canals, ${ }^{11,12,17}$ but studies on the filing of the safety zone are still lacking.

Thus, because of the importance of anticurvature filing in root canal preparation and the accidents that may occur during this procedure, the objective of this study was to propose adequate planning for effective anticurvature filing with reduced loss of dentin structure, taking into account pre-determined anatomical points on teeth to establish directions for proper implementation of the technique.

\section{Methodology}

Two hundred digital periapical radiographs of human molar teeth were selected (gathered following informed consent according to a protocol approved by the Committee for Ethics in Research of the Universidade Federal de Uberlândia: \#164.043). The radiographs were obtained from records of patients treated at a private practice office. All radiographs were taken by the same operator using Spectro 70X (Dabi Atlante, Ribeirão Preto, Brazil), a conventional x-ray unit associated with an intraoral digital x-ray sensor, CDR 2000 (Schick Technologies Inc., New York, USA).

The inclusion criteria consisted of radiographs of first and second maxillary and mandibular molars obtained in bucco-lingual incidence without distortions. Only radiographs from mandibular molars presenting overlapping of the mesial roots in which a single radiolucent root canal image was observed were considered. The radiographs were then divided into two groups $(n=100)$ : $M X$, maxillary molars; and MD, mandibular molars. The mesiobuccal roots were considered in the MX group and the mesial roots in the MD group.

Image editing software, Adobe Photoshop 7.0 (Adobe Systems Inc., San Jose, USA), was used for marking the canal paths of the roots and the pre-determined anatomical points: IC, initial path of the root canal; CPP, critical penetration point of the file in the canal; MP, maximum canal projection; and PL, periodontal root limit (Figure 1A). The CPP point was evidenced by a straight line from the initial canal path up to the displacement of its long axis, characterizing the beginning of the canal curvature (Figure 1A). The MP point was located at the central point of the most convex part of the canal path, evidenced by a vertical line tangent to this point (Figure 1A). The PL point was characterized by a horizontal line from the root canal opening up to the lateral periodontal region (Figure 1A). 

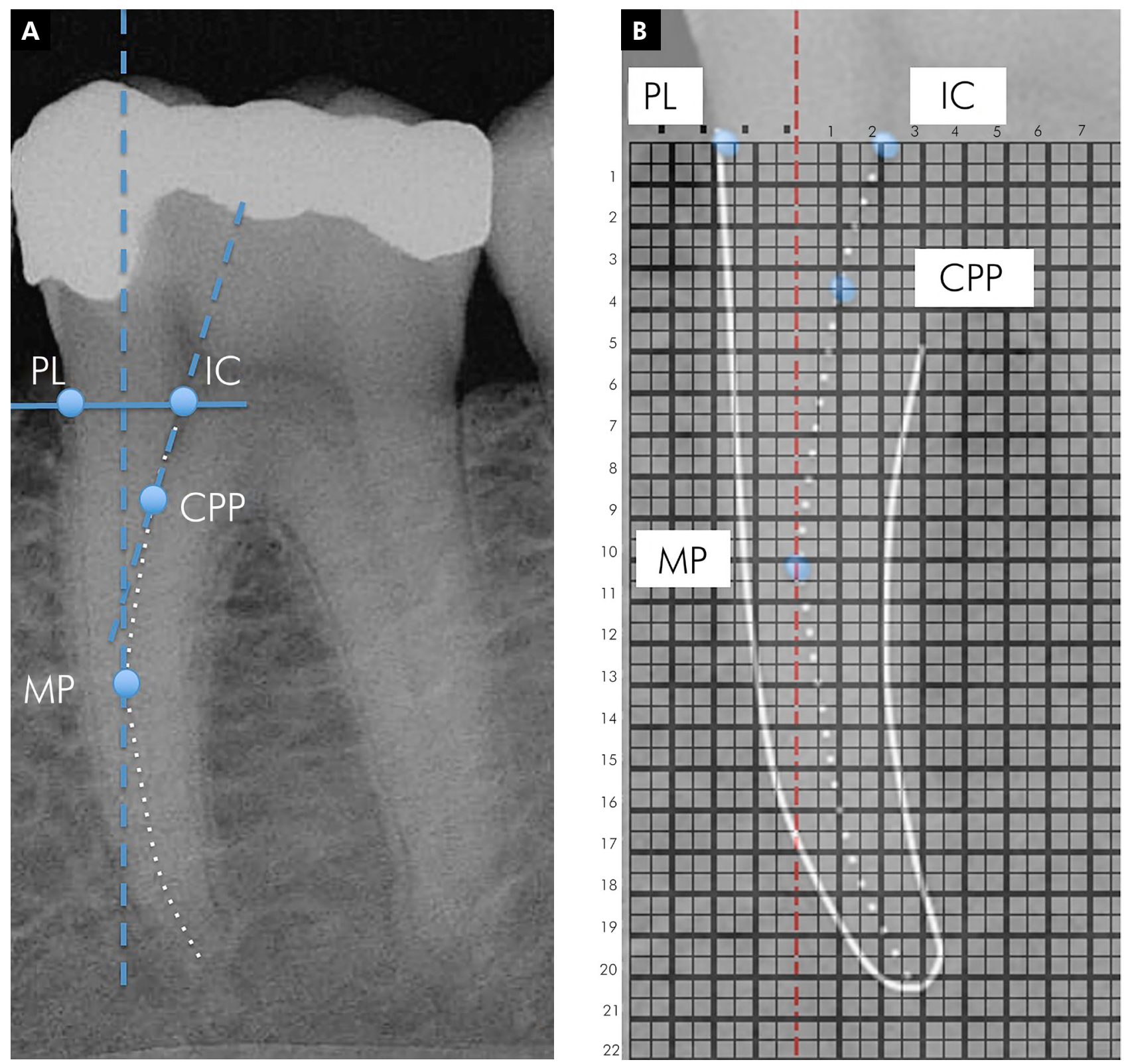

Figure 1. (A) Determination of canal path and anatomical points (IC, CPP, PL, and MP); (B) Grid overlapping the mesial root (*red line indicating zero point).

To acquire numerical data for the marked points, we transferred the images to Microsoft Excel 2010 (Microsoft Corp., Washington, USA), in which a millimeter grid was set over the images. The grid had $0.5-\mathrm{mm}$ markings, totaling $15 \mathrm{~mm}$ in length and $22 \mathrm{~mm}$ in height. The grid was set over the images with the IC and PL points in the vertical zero limit and the MP point in the horizontal zero limit (Figure 1B). The distances $(\mathrm{mm})$ between the anatomical points were then obtained in the horizontal $(\mathrm{H})$ and vertical $(\mathrm{V})$ directions (Figures 2A and 2B).

\section{Simulation of the anticurvature filing}

Two different anticurvature filing protocols were simulated in the present study: SF, straight access filing; and $\mathrm{CF}$, conservative filing. $\mathrm{SF}$ was simulated through a vertical line drawn from the crown to the maximum canal projection in the vertical plane (MP) (Figure 3A), 

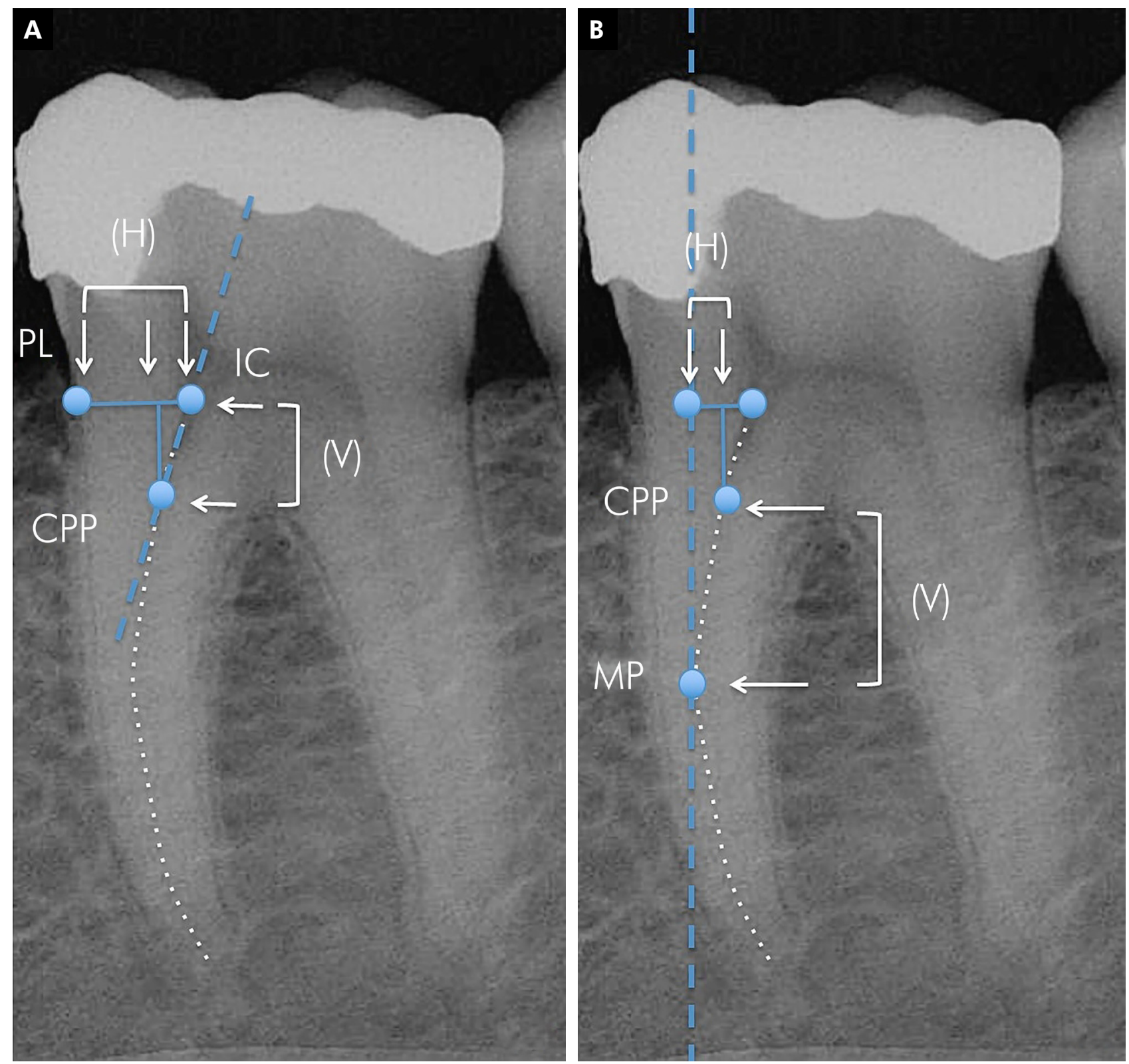

Figure 2. Measurements of the distances between the points: (A) IC-CPP and IC-PL and (B) IC-MP and CPP-MP, in vertical (V) and horizontal $(\mathrm{H})$ directions.

intended to allow for straight access of files to the root canal. CF was simulated to allow for straight access of endodontic instruments through a vertical straight line parallel to the mesial root wall, connecting the occlusal limit of coronal access (OL) to the MP point (Figure 3B). The area of dentin removal in the root (DRR), for both filing protocols, was represented by a triangle formed by the IC, MP, and a point determined by the intersection between IC and PL, generated from the positioning of the file after determination of the anatomical points for the anticurvature filing (Figure 3). The area of dentin removal in the crown (DRC) for both filing protocols was represented by a quadrangle or a triangle connecting the vertical line to the OL and IL points (Figure 3). The DRR, DRC areas, and the remaining dentin distance after filing simulation were evaluated and quantified in the mesial surface (RSZ, safety zone) and distal surface (RDZ, danger zone) with Image J (NIH, Bethesda, USA). 

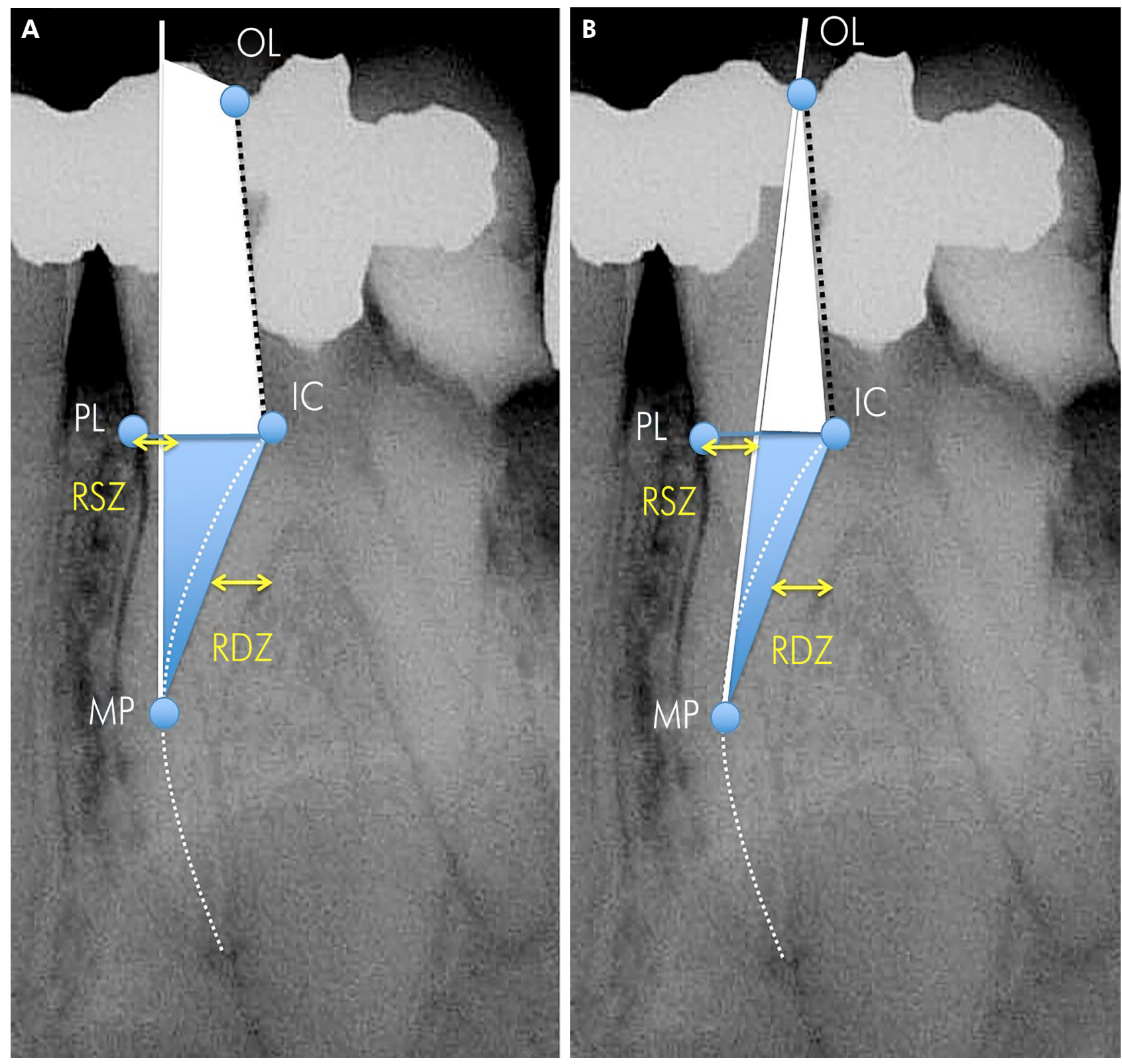

Figure 3. (A) Simulated straight access filing (SF); (B) Simulated conservative filing (CF). Remaining dentin in the safety (RSZ) and danger (RDZ) zones ('yellow arrows), and areas of dentin removal in the root (*blue triangle) and in the crown ("white area).

The collected data were subjected to theShapiro-Wilk and Levene tests for the determination of normality and homogeneity. The comparison between the groups before and after filing simulation was made by thet-test and the Mann-Whitney test. The data obtained after filing simulation were analyzed by one-way analysis of variance (ANOVA), with subdivided parcels and Tukey's test for comparisons of both filing protocols inside each group. The Spearman Rank Order Correlation test was used to evaluate the correlation between the data obtained after the filing simulations. All tests were performed at the $5 \%$ level of significance with SigmaPlot v.12.0 (Systat Software Inc., Chicago, USA).

\section{Results}

The data from the measurements of the distances verified between the anatomical points before the anticurvature filing simulation (Figures 2A and 2B) 
are shown in Table 1 . In between-group comparisons, significant differences were detected $(p<0.001)$, with the MX group showing higher values in the horizontal direction, while the MD group exhibited higher values in the vertical direction.

The data from the measurements obtained after the anticurvature filing simulation relative to the filing area and remaining dentin (Figure 3) are shown in Table 2. For between-group comparisons, significantly higher values were found for the MX group $(p<0.001)$. When the filing protocols were compared, significantly

Table 1. Median distance values between the anatomical points in the vertical $(\mathrm{V})$ and horizontal $(\mathrm{H})$ directions related to groups of maxillary molars (MX) and mandibular molars (MD).

\begin{tabular}{lccc}
\hline $\begin{array}{l}\text { Distances between the } \\
\text { anatomical points }(\mathrm{mm})\end{array}$ & $M X$ & $M D$ & p-value \\
\hline IC-MP (H) & 2.0 & 1.6 & $<0.001$ \\
IC-MP (V) & 6.0 & 7.5 & $<0.001$ \\
IC-CPP (H) & 1.7 & 1.5 & $<0.001$ \\
IC-CPP (V) & 4.0 & 5.3 & $<0.001$ \\
CPP-MP (H) & 0.2 & 0.1 & 0.152 \\
CPP-MP (V) & 1.7 & 2.0 & 0.244 \\
IC-PL (H) & 4.0 & 3.5 & $<0.001$ \\
\hline
\end{tabular}

*IC: Initial path of the root Canal; MP: Maximum canal Projection; CPP: Critical Penetration Point; PL: Periodontal root Limit.

Table 2. Median values obtained after anticurvature filing protocols (SF, straight access filing; CF, conservative filing) related to the thickness $(\mathrm{mm})$ of the root dentin remaining in the safety zone (RSZ) and danger zone (RDZ) and the areas (mm2) of dentin removal in the root (DRR) and in the crown (DRC).

\begin{tabular}{|c|c|c|c|c|}
\hline \multicolumn{5}{|c|}{ Median values } \\
\hline Filing protocol & Region & $M X$ & MD & $p$-value \\
\hline SF & RSZ (mm) & $2.0^{\mathrm{Aa}}$ & $2.0^{\mathrm{Aa}}$ & 0.066 \\
\hline \multirow[t]{2}{*}{ CF } & $\mathrm{RSZ}(\mathrm{mm})$ & $2.3^{B a}$ & $2.0^{\mathrm{Bb}}$ & 0.004 \\
\hline & $p$-value & $<0.001$ & $<0.001$ & \\
\hline SF & RDZ (mm) & $1.5^{\mathrm{Aa}}$ & $1.5^{\mathrm{Aa}}$ & 0.340 \\
\hline \multirow[t]{2}{*}{ CF } & $\mathrm{RDZ}(\mathrm{mm})$ & $1.5^{\mathrm{Aa}}$ & $1.5^{\mathrm{Aa}}$ & 0.340 \\
\hline & $p$-value & 1.000 & 1.000 & \\
\hline SF & $\mathrm{DRR}\left(\mathrm{mm}^{2}\right)$ & $5.6^{\mathrm{Aa}}$ & $5.2^{\mathrm{Aa}}$ & 1.000 \\
\hline \multirow[t]{2}{*}{$\mathrm{CF}$} & $\mathrm{DRR}\left(\mathrm{mm}^{2}\right)$ & $4.8^{\mathrm{Ba}}$ & $4.3^{\mathrm{Ba}}$ & 0.224 \\
\hline & $p$-value & $<0.001$ & $<0.001$ & \\
\hline SF & $\mathrm{DRC}\left(\mathrm{mm}^{2}\right)$ & $12.1^{\mathrm{Aa}}$ & $8.9^{\mathrm{Ab}}$ & 0.002 \\
\hline \multirow[t]{2}{*}{ CF } & $\mathrm{DRC}\left(\mathrm{mm}^{2}\right)$ & $8.6^{\mathrm{Ba}}$ & $6.6^{\mathrm{Bb}}$ & $<0.001$ \\
\hline & $p$-value & $<0.001$ & $<0.001$ & \\
\hline
\end{tabular}

*Different capital letters indicate significant differences between the filing protocols (vertical). Small letters indicate significant differences between the groups (horizontal) $(p<0.05)$. higher values were found for theSF protocol $(p<0.001)$, except for the dentin remaining in the safety zone.

The correlations between the data verified before and after the anticurvature filing simulation are presented in Table 3. The distances between the anatomical points showed negative correlation to the dentin remaining in the safety and danger zones in the root, and positive correlation to the areas of dentin removal in the root (DRR) for both experimental groups.

\section{Discussion}

The anticurvature filing represents an important technique for the endodontic treatment of curved canals, ${ }^{3}$ though some care with the direction of the instruments is required to prevent weakening of root dentin. Some anatomical parameters should be taken into account in the planning of anticurvature filing, since anatomy imposes limits that, if correctly observed, will prevent excessive filing of the root structure.

Taking into account the anatomical points related to the root canal path, as in the present study, can allow for adequate planning of the anticurvature filing. The critical penetration point (CPP) represents the initial curvature of the root canal path, in which endodontic instruments find the first obstacle during canal penetration. This region can be located at any area of the canal that includes the initial canal path (IC) and its maximum projection (MP), in the mesiodistal (MD) and buccolingual (BL) directions of the root. Its location is significant for the planning of anticurvature filing and, in this study, was a factor in the results observed in the simulations performed. The CPP location was observed in the cervical third in $57 \%$ of cases for the MX group and in $68 \%$ for the MD group, making its displacement necessary to the region of maximum projection of the canal, allowing for approximation of the apical region. The closer the CPP is to the root apex, the easier it will be to keep the original root canal path, due to the greater flexibility at the end of the file. The literature recommends that cervical pre-flaring performed before canal instrumentation should allow for free straight access of files up to the apex or to the initial canal curvature. ${ }^{1,5,6,7,9,10,17}$ Following this precept, file access at mesiobuccal canals in maxillary molars and 
Table 3. Correlation of anatomical points and thickness of the remaining root dentin in the safety zone (RSZ), danger zone (RDZ), and area of dentin removal in the root (DRR) in the vertical $(V)$ and horizontal $(H)$ directions.

\begin{tabular}{|c|c|c|c|c|}
\hline Correlation & MX group & $p$-value & MD group & $p$-value \\
\hline IC-MP $(H) \times R S Z$ & -0.4 & $<0.001$ & -0.5 & $<0.001$ \\
\hline IC-MP $(V) \times R S Z$ & -0.3 & 0.002 & -0.2 & 0.044 \\
\hline IC-MP $(H) \times R D Z$ & -0.4 & $<0.001$ & -0.4 & $<0.001$ \\
\hline IC-MP $(V) \times R D Z$ & -0.5 & $<0.001$ & -0.4 & $<0.001$ \\
\hline IC-MP $(H) \times$ DRR & 0.8 & $<0.001$ & 0.8 & $<0.001$ \\
\hline IC-MP $(V) \times D R R$ & 0.8 & $<0.001$ & 0.7 & $<0.001$ \\
\hline IC-CPP $(\mathrm{H}) \times \mathrm{DRR}$ & 0.7 & $<0.001$ & 0.7 & $<0.001$ \\
\hline IC-CPP $(V) \times D R R$ & 0.3 & 0.009 & 0.2 & 0.070 \\
\hline MP-CPP $(\mathrm{H}) \times$ DRR & 0.6 & $<0.001$ & 0.6 & $<0.001$ \\
\hline MP-CPP $(\mathrm{V}) \times \mathrm{DRR}$ & 0.6 & $<0.001$ & 0.7 & $<0.001$ \\
\hline IC-PL x DRR & 0.3 & 0.007 & 0.3 & $<0.001$ \\
\hline
\end{tabular}

mesial canals in mandibular molars would occur only up to the initial canal curvature, represented by the CPP point in this research. The extent of anticurvature filing at only this point may promote the formation of steps in the canal path. Thus, filing extension until the maximum projection of the canal (MP) is suggested, since the initial barrier would be overcome thereby.

The maximum canal projection (MP) represents the maximum vertical limit for the orientation of filing on the outer walls of the root canal. The MP point was not coincident with the initial canal curvature (CPP) in $100 \%$ of canals from the MX group and in 99\% from the MD group, being located at an average of $2.27 \mathrm{~mm}$ after the CPP. Following anticurvature filing simulation, the CPP was displaced to the $\mathrm{MP}$, being located in the medium third in $89 \%$ of canals from the MD group and in $85 \%$ from the MX group. This represents the displacement of the initial canal curvature from one canal third to another. Additionally, the greater the distance between CPP and MP points, the higher the root dentin area removed in the anticurvature filing, for both the horizontal and vertical directions.

The occlusal limit point (OL) located at the tooth crown is the limit for the anticurvature filing in the occlusal regions. This limit was considered after complete removal of the roof of the pulp chamber and pulp horns in the crown opening. A straight line connecting the OL and MP points of the canal path determined the positioning and inclination of the file after anticurvature filing (Figure 3B). In the simulations, it was observed that the file inclination presented various angles, depending on the characteristics of each tooth. This fact demonstrates that it is not always necessary for canals to be accessed with files at a vertical straight line after anticurvature filing, as suggested by some studies. ${ }^{1,5,6,7,9,10,17}$

The straight access of files in a vertical line after anticurvature filing simulation was possible in only $29 \%$ of mesiobuccal canals from maxillary molars and in $46 \%$ of mesial canals from mandibular molars. In the other cases, reaching straight file access would require excessive removal of dentin from coronal and cervical third regions, which can lead to weakening of tooth structure, thus increasing the risk of vertical root fractures (Figure 3A).

In comparison of wear produced by the two anticurvature filing protocols for both experimental groups, the area of dentin removal at the danger zone (RDZ) was found to be unaffected. However, the different filing protocols influenced the area of dentin removal in the crown and root in the safety zone (RSZ). The straight access filing (SF) promoted increased dentin removal compared with the conservative filing (CF), showing total wear (crown and root) superior to $36 \%$ of the MX group and to $20 \%$ of the MD group. Since both filing protocols moved the critical penetration point (CPP) to the maximum canal projection (MP) and produced similar wear at the danger zone (RDZ), the conservative anticurvature filing $(\mathrm{CF})$ protocol seems the best option, because it 
produces less removal of crown and root dentin in the safety zone (RSZ), reducing the risk of fractures by tooth weakening.

Despite the increased area of dentin removal observed for the MX group compared with the MD group with the same anticurvature filing protocol, the dentin remaining in the two zones of the root was similar for both groups. The influence of anatomical differences between the groups was detected only for dentin removal in the crown, reinforcing the assertion that there is no need to perform different anticurvature filing techniques for maxillary and mandibular molars, particularly if the anatomical points presented in this study are taken into consideration.

The distance between the initial canal path and the maximum projection (IC-MP) influenced the remaining dentin thickness after the anticurvature filing simulation. It was observed that the greater the distance in the vertical and horizontal directions, the lower the thickness of the remaining dentin following the procedure in the danger and safety zones of the root. The increased distance between the IC-MP points is of clinical concern in these two root zones. In the safety zone, this thickness presents no perforation risk, but may weaken dentin by excess filing in an attempt to obtain vertical straight access of files. In the danger zone, the possibility of perforation with periodontal exposure was observed more frequently in the mesiobuccal roots of maxillary molars, which presented a $0.6-\mathrm{mm}$ remaining thickness at the danger zone after anticurvature filing simulation.

Therefore, it is suggested that when a great distance between the initial path and its maximum projection (IC-MP) is observed radiographically, the anticurvature filing should be initiated with manual or rotary files of reduced conicity to increase the control of file orientation and filing in the danger zone. In addition, excessive filing in the safety zone during attempts at straight canal access should be avoided because, in these cases, the file will present distal inclination.

The research design of this study presents some intrinsic limitations, since it was conducted during simulations of different anticurvature filing protocols in digital radiographs of clinically obtained maxillary and mandibular molars. Future studies that overcome these limitations with laboratory and clinical evaluations of the parameters presented will be of benefit.

\section{Conclusion}

The radiographic location of the critical penetration point (CPP) and its relation to the other anatomical points described are essential for maintaining the original root canal path and for planning the anticurvature filing.

Conservative anticurvature filing seems the best option, since it may remove reduced amounts of crown and root dentin and, even with anatomical differences, can be performed in the same manner for maxillary and mandibular molars.

Correlation was observed between the distance of the anatomical points and the amount of root dentin removal after the anticurvature filing.

\section{Acknowledgments}

The authors would like to acknowledge to the Fundação de Amparo à Pesquisa do Estado de Minas Gerais (FAPEMIG) for the financial support of the project and Dr. Cristiane M. Caram for the support in the acquisition of digital radiographies. 


\section{References}

1. Goerig AC, Michelich RJ, Schultz HH. Instrumentation of root canals in molars using the step-down technique. J Endod. 1982 Dec;8(12):550-4.

2. Hülsmann M, Peters OA, Dummer PMH. Mechanical preparation of root canals: shaping goals, techniques and means. Endod Topics. 2005 Aug;10(1):30-76.

3. Abou-Rass M, Frank AL, Glick DH. The anticurvature filing method to prepare the curved root canal. J Am Dent Assoc. 1980 Nov;101(5):792-4.

4. Constante IGT, Davidowicz H, Barletta FB, Moura AAM. Study of the areas and thicknesses of mesiobuccal root canals prepared by three endodontic techniques. Braz Oral Res. 2007 Apr-Jun;21(2):118-26.

5. Iqbal A, Akbar I, AL-Omiri MK. An in vivo study to determine the effects of early preflaring on the working length in curved mesial canals of mandibular molars. J Contemp Dent Pract. 2013 Mar;14(2):163-7.

6. Tan BT, Messer HH. The effect of instrument type and preflaring on apical file size determination. Int Endod J. 2002Sep;35(9):752-8.

7. Pécora JD, Capelli A, Guerisoli DMZ, Spanó JCE, Estrela C. Influence of cervical preflaring on apical file size determination. Int Endod J. 2005 Jul;38(7):430-5.

8. Silveira LFM, Silveira CF, Castro LAS, César Neto JB, Martos J. Crown-down preflaring in the determination of the first apical file. Braz Oral Res. 2010 Apr-Jun;24(2):153-7.

9. Camargo EJ, Zapata RO, Medeiros PL, Bramante CM, Bernardineli N, Garcia RB, et al. Influence of preflaring on the accuracy of length determination with four electronic apex locators. J Endod. 2009 Sep;35(9):1300-2.
10. Ehrhardt IC, Zuolo ML, Cunha RS, De Martin AS, Kherlakian D, Carvalho MC, et al. Assessment of the separation incidence of Mtwo files used with preflaring: prospective clinical study. J Endod. 2012 Aug;38(8):1078-81.

11. Wu M-K, Van der Sluis LWM, Wesselink PR. The risk of furcal perforation in mandibular molars using Gates-Glidden drills with anticurvature pressure. Oral Surg Oral Med Oral Pathol Oral Radiol Endod. 2005 Mar;99(3):378-82.

12. Akhlaghi NM, Kahali R, Abtahi A, Tabatabaee S, Mehrvarzfar P, Parirokh M. Comparison of dentine removal using V-taper and K-Flexofile instruments. Int Endod J. 2010 Nov;43(11):1029-36.

13. Mahran AH, AboEl-Fotouh MM. Comparison of effects of ProTaper, HeroShaper, and Gates Glidden burs on cervical dentin thickness and root canal volume by using multislice computed tomography. J Endod. 2008 Oct;34(10):1219-22.

14. Sanfelice CM, Costa FB, Só MVR, Vier-Pelisser F, Bier CAS, Grecca FS. Effects of four instruments on coronal pre-enlargement by using Cone Beam computed tomography. J Endod. 2010 May;36(6):858-61.

15. Ree M, Schwartz RS. The endo-restorative interface: current concepts. Dent Clin North Am. 2010 Apr;54(2):345-74.

16. Raiden G, Koss S, Costa L, Hernández JL. Radiographic measurement of residual root thickness in premolars with post preparation. J Endod. 2001 Apr;27(4):296-8.

17. Coutinho-Filho T, De-Deus G, Gurgel-Filho ED, Rocha-Lima AC, Dias KRC, Barbosa CA. Evaluation of the risk of a stripping perforation with Gates-Glidden drills: serial versus crowndown sequences. Braz Oral Res. 2008 Jan-Mar;22(1):18-24. 